\title{
A Special Issue on Stem Cell Research
}

Cell Research (2007) 17: 1-2. doi: 10.1038/sj.cr.7310142; published online 15 January 2007

Stem cells possess the remarkable ability of extensive self-renewal and differentiation into specific cell lineages, and they play essential roles in development and adult tissue homeostasis. Due to their critical importance in normal physiology and the promise for use in regenerative medicine to treat a variety of diseases, stem cells have attracted extensive research interest in recent years. As China's premium international journal with a broad scope in cell and molecular biology, Cell Research has witnessed more and more submissions on stem cell research. Indeed, along with the traditional strengths of the journal in molecular immunology, cancer biology, and plant molecular physiology, stem cell research has gradually and naturally evolved into a new growth point of Cell Research. Reflecting the growing interest of both our readers and authors in this exciting and expanding field, we are pleased to present this Special Issue on Stem Cell Research.

The life of a stem cell in vivo is governed by both its own intrinsic properties and the communications with its microenvironment (the stem cell niche). Dániel Kirilly and Ting Xie review the stem cell community in Drosophila ovary, which has served as an excellent model system to study fundamental aspects of stem cell biology, including regulation of stem cells by niche signals; while Scheffer Tseng and colleagues update us on studies of niche regulation of a unique group of stem cells in the eye, the corneal epithelial stem cells. In a Research Article, Jinhua Wen, Lingsong Li, and coworkers used neural stem cells differentiated from mouse embryonic stem cells as a model system to study the effect of putative niche signals, and they report mechanistic insights on the collaborative actions of two such signals, bFGF and VEGF.

Compared with adult stem cells which are usually restricted in their lineage potentials, embryonic stem (ES) cells are capable of differentiating into cells of all three germ layers (pluripotency). Understanding the molecular mechanisms underlying ES cell (especially human ES cell) pluripotency and differentiation is of obvious importance to stem cell therapy. Guangjin Pan and James Thomson review the transcriptional networks critical for the maintenance of ES cell pluripotency, involving Nanog and other transcription factors (such as Oct4 and Sox2); and Gen-Sheng Feng discusses the physiological role of Shp2, a signaling phosphatase, in regulation of ES cell differentiation. In their Research Articles, Linzhao Cheng and coworkers report important progress in culturing and clonal growth of human ES cells using engineered human feeder cells expressing certain Wnt ligands; while Liu Wang, Fanyi Zeng, Qi Zhou and colleagues established a large number of mouse ES cell lines by nuclear transfer or sequential nuclear transfer and examined the developmental potential of the corresponding embryos.

The study of different stem cell populations often offers unique insights into the respective physiological or pathological processes. Yong Zhao and colleagues discuss evidence for common thymic progenitor cells giving rise to both thymic cortical and medullary epithelial cells which play critical roles in $\mathrm{T}$ cell 
development. Ana Teixeira, Joshua Duckworth and Ola Hermanson review recent advances in the use of polymers and derived biomaterials to control neural stem cell properties and delivery. Finally, Yibin Kang and colleagues update us on cancer stem cells (CSCs) and propose an intriguing model of cancer progression whereby CSCs play a central role in both tumorigenesis and metastasis.

In short, we are very excited to be bringing to our readers this outstanding collection of Review and Research Articles, and would like to thank all of our authors for their contributions and support to Cell Research. We are also especially grateful to Drs Duanqing Pei, Linzhao Cheng, Ting Xie, and Yun-Bo Shi, who have served as Editors or Guest Editors for this Special Issue. Their great efforts have been instrumental to the successful assembly of this special collection. The publication of this Special Issue not only brings cutting edge scientific information to our readers, but also clearly signals that Cell Research will continue to have a strong interest in publishing quality papers in stem cell research, a fast-changing and exciting area of today's biology. Finally, we wish that all of our readers and authors have a happy and productive new year; and please, sit back and enjoy this Special Issue!

\title{
Dangsheng Li, PhD
}

Deputy Editor-in-Chief, Cell Research

\author{
AND \\ Gang Pei, PhD \\ Editor-in-Chief, Cell Research
}

